\title{
Erratum
}

\section{Facile Synthesis of Fluorinated 1-Desazapurines}

Viktor O. Iaroshenko, * Dmitri V. Sevenard, Dmitriy M. Volochnyuk, * Yan Wang, Alexander Martiloga, Andrei O. Tolmachev Synthesis 2009, 1865.

The advance online publication (e-First) version of this article contained the incorrect structural representation for AIRs-riboside 1 in Scheme 1.

Further, the ${ }^{1} \mathrm{H}$ NMR data for compound $\mathbf{1 2 b}$ have been corrected.

These mistakes have been corrected for both the current online version and the print publication. 